Journal of Advanced Research in Fluid Mechanics and Thermal Sciences

Journal homepage: www.akademiabaru.com/arfmts.html ISSN: 2289-7879

\title{
Stability Convection in a Couple Stress Fluid Saturated in an Anisotropic Porous Medium with Internal Heating Effect
}

\author{
Nadia Diana Mohd Rusdi ${ }^{1}$, Nor Fadzillah Mohd Mokhtar ${ }^{1,2, *}$, Norazak Senu ${ }^{1,2}$, Siti Suzilliana Putri \\ Mohamed Isa ${ }^{1,2}$ \\ 1 Laboratory of Computational Sciences and Mathematical Physics, Institute for Mathematical Research (INSPEM), Universiti Putra Malaysia, \\ Malaysia \\ 2 Department of Mathematics, Faculty of Science, Universiti Putra Malaysia, Malaysia
}

\section{ARTICLE INFO}

\section{Article history:}

Received 2 June 2020

Received in revised form 15 August 2020

Accepted 28 August 2020

Available online 16 October 2020

\section{Keywords:}

Convection; Anisotropic; Internal heating; Couple-stress fluid

\section{ABSTRACT}

\begin{abstract}
Internal heating effect with stability convection in a couple stress fluid saturated in an anisotropic porous medium has been studied numerically using linear stability analysis. The presence of internal heating on couple stress fluid in an anisotropic porous medium heated from below has been verified. The momentum equation and Boussinesq approximation is used for the density variation in the porous medium. By using Chebyshev Tau method numerically, the eigenvalue problems of the perturbed state were obtained from a normal mode analysis. The effect of the Rayleigh number, internal heat source and anisotropy parameter has been shown graphically. The critical Rayleigh number also has been obtained and plotted on the system. From the result, it is found that the mechanical anisotropy parameter and internal heating effect destabilized the system while couple stress fluid and thermal anisotropy parameter help in stabilizing the system.
\end{abstract}

\section{Introduction}

Problem of fluid with couple stress in a porous medium attracted researcher widely. Many researchers also interested in doing research on internal heating relates to their field of studies but only several researchers considered it with couple stress fluid. Numerous applications include system of electro-chemistry, geophysical system and fossil fuels for the studies. Internal heating is from the heat source or heat energy for outer space bodies which can caused by radioactive or decay materials and nuclear atoms to keep it warm and active described by Bhadauria [1]. Couple stress fluid is the kinematic level that leads to velocity field with the presence of the isolation of the fluid Stokes [2].

Previous researcher concerned one of the studies related to fluid of the couple stress with double convection of diffusive analytically and linear stability analysis is used in a horizontal saturated

\footnotetext{
* Corresponding author.

E-mail address: norfadzillah.mokhtar@gmail.com
}

https://doi.org/10.37934/arfmts.76.2.7584 
anisotropic of porous Gaikwad and Kouser [3]. Effects of Soret with Dufour are considerable importance for their studies. Furthermore, Malashetty and Kollur [4] has been investigating the same method but the difference is double diffusive convection in which has been included in the studies, which is heated and salted from below. Sharma et al., [5] had done their study on fluid related to couple stress when the rotation and magnetic field that considered stable vertical for both effects. Pranesh and Bawa [6] has considered the Rayleigh-Benard which is related to convection with the effects of internal heat generation and magnetic field in nanofluid while Nield and Kuznetsov [7] also using internal heating with the presence of nanofluid related to the convection. Linear stability was applied in the studies. New study from Storesletten and Rees [8] investigated the combination of both effects which is internal heat generation and inclination anisotropy for instability linear.

Anisotropic porous medium has become one of famous research area. Srivastava et al., [9] has been using anisotropic porous medium in their research add together internal heating effects and viscosity liquid variable under G-jitter during the process of heat transport. In addition, the source of internal heating and the existence of forced time with gravity field has been studied narrowly in the research. The beginning of double diffusive convection has been studied by Malashetty and Heera [10] with the effect of rotation and anisotropic porous layer by using linear and weak nonlinear theory. With the same theory, Bhadauria et al., [11] have carried out rotating anisotropic porous layer with internal heat source in linear, nonlinear in the thermal instability.

The aim of this paper to study the internal heating effect and couple stress fluid in an anisotropic porous medium. Our intend in order to find the critical Rayleigh number in a horizontal porous medium with upper free conducting boundary conditions, respectively. Further, the eigenvalue problem will be calculated in QZ algorithms by using Chebyshev Tau method.

\section{Methodology}

A fluid of couple stress with horizontal anisotropic porous medium restricted to two horizontal planes which is parallel to one another at $z=0, z=d$, the depth, $d$ which heated with internal heat source from below, were considered as shown in Figure 1 . In addition, the gravity of the system is moving downward, $g=(0,0,-g)$ and stable adverse difference of temperature, $\Delta T=T_{s}-$ $T_{v}$ where $T_{s}$ and $T_{v}$ is the constant value of lower and upper temperature. The porous medium in mechanical properties and thermal properties are expected to be anisotropic in the horizontal direction. However, the density porous will consider an effect which can depend linearly on temperature. All these assumptions, which given by

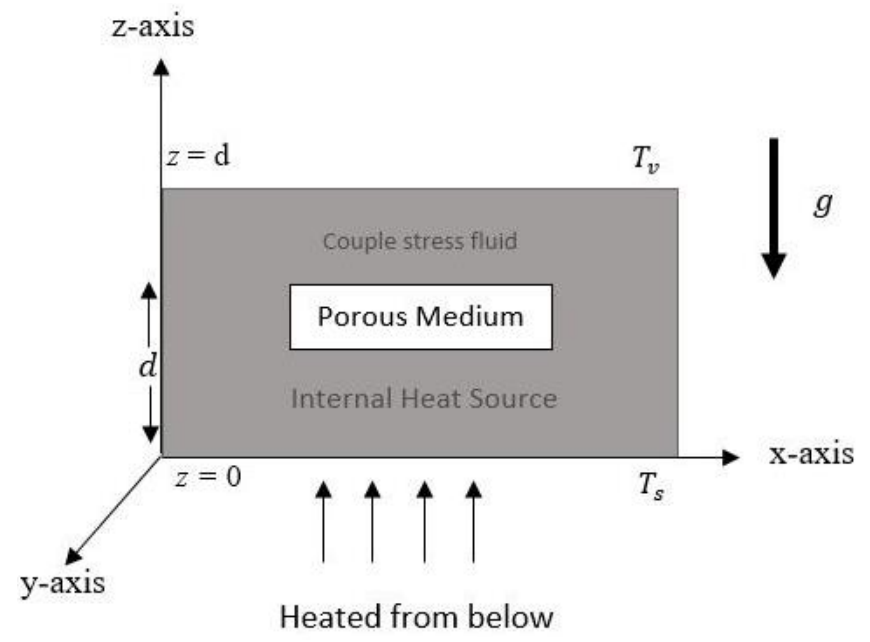

Fig. 1. Geometry of the Problem 
From Figure 1, Boussinesq approximation from the perturbation quantities are given by

$\nabla \cdot \vec{w}=0$

$\frac{\rho_{0}}{\phi} \frac{\partial \vec{w}}{\partial t}+\frac{\mu}{\vec{K}} \cdot \vec{w}+\nabla p-\rho g+\left(\mu-\mu_{c} \nabla\right) \vec{w}=0$,

$\gamma \frac{\partial T}{\partial t}+(\vec{w} \cdot \nabla) T-Q\left(T-T_{0}\right)-\kappa_{T z} \nabla^{2} T$.

$\rho=\rho_{0}\left[1-\alpha\left(T-T_{0}\right)\right]$

The thermal of boundary conditions is given as

$T=T_{0}+\Delta T, z=0$,

$T=T_{0}, z=d$

where $\vec{w}=(u *, v *, w *)$ is the velocity vector, the pressure denoted by $p, \phi$ is the porosity, $\vec{K}=$ $K_{x}\left(\vec{\imath} i^{*}+\vec{\jmath} j^{*}\right)+K_{z}\left(\vec{k} k^{*}\right)$ is the permeability tensor with the dynamic viscosity by symbol $\mu, \mu_{c}$ is the couple stress viscosity of the fluid, $Q$ is the source of heat, $\gamma$ is the heat capacity, $T$ are the heat temperature, $\kappa_{T z}$ is the vertical thermal diffusivity, $\rho_{0}$ and $\alpha$ are the reference density and thermal expansion coefficient respectively. First, the basic state fluid is assumed not moving or at rest, defined by

$\vec{w}_{b}=(0,0,0), \quad T=T_{b}(z), \quad p=p_{b}(z), \rho=\rho_{b}(z)$

where the basic is assumed to be the subscript of $b$ which can also be written in the following equations given when we substitute Eq. (7) into Eq. (1)-(6).

$\frac{\mathrm{d} p_{b}}{\mathrm{~d} z}=-\rho g, \quad \frac{\mathrm{d}^{2} T_{b}}{\mathrm{~d} z}=0, \quad \rho_{b}=\rho\left[1-\alpha\left(T_{b}-T_{0}\right)\right]$,

$\kappa_{T} \frac{\mathrm{d}^{2}\left(T_{b}-T_{0}\right)}{\mathrm{d} z^{2}}+Q\left(T_{b}-T_{0}\right)=0$,

The condition state of temperature in Eq. (9) as follows

$T_{b}=T_{0}+\Delta T \frac{\sin \sqrt{\frac{Q d^{2}}{\kappa_{T_{z}}}}\left(1-\frac{z}{d}\right)}{\sin \sqrt{\frac{Q d^{2}}{\kappa_{T_{z}}}}}$,

subject to boundary conditions Eq. (5) and Eq. (6). Next, the infinitesimal perturbation was superposed for the basic state which satisfy the equations

$\vec{w}=\vec{w}_{b}+w^{\prime}, \vec{T}=\vec{T}_{b}+T^{\prime}, \vec{p}=\vec{p}_{b}+p^{\prime}, \rho=\vec{\rho}_{b}+\rho^{\prime}$, 
where the prime shows the perturbation quantities. Pressure term will be eliminated by using the infinitesimal perturbations with the curl curl formula on momentum equations from the porous medium. The resulting equations will be dimensionlized by transforming the following transformation

$$
\begin{aligned}
& t=\frac{\gamma \mathrm{d}^{2} t^{*}}{\kappa_{T z}}, \quad T^{\prime}=(\Delta T) T^{*}, \quad\left(x *^{\prime}, y *^{\prime}, z *^{\prime}\right)=\left(x *^{*} \mathrm{~d}, y *^{*} \mathrm{~d}, z *^{*} \mathrm{~d}\right),\left(u *^{\prime}, v *^{\prime}, w *^{\prime}\right)= \\
& \left(\frac{\kappa_{T z} u *^{*}}{\mathrm{~d}}, \frac{\kappa_{T z} v *^{*}}{\mathrm{~d}}, \frac{\kappa_{T z} w *^{*}}{\mathrm{~d}}\right),
\end{aligned}
$$

Hence, the dimensionless resulting equations will depict

$$
\begin{aligned}
& {\left[\frac{1}{V a} \frac{\partial}{\partial t} \nabla_{h}^{2}+\left(\nabla_{h}^{2}+\frac{1}{\xi} \frac{\partial^{2}}{\partial z^{* 2}}\right)\left(1-C \nabla_{h}^{2}\right)\right] w^{*}-R a_{T} \nabla_{h}^{2} T^{*}=0,} \\
& {\left[\gamma \frac{\partial}{\partial t^{*}}-\eta \nabla_{h}^{2}-\frac{\partial^{2}}{\partial z^{* 2}}+\vec{u}^{*} \cdot \nabla\right] T^{*}-[Q((1-2 z)-1)] w^{*}=0,}
\end{aligned}
$$

where $V a=\phi \frac{P r}{D a}$ is the Vadasz number and $D a=\frac{K_{z}}{d^{2}}$ is the Darcy number, $\operatorname{Pr}=\frac{\mu}{\rho_{0} \kappa_{T z}}$ is the Prandtl number, $R a=\frac{\alpha g \Delta T d K_{z}}{v \kappa_{T z}}$ is the thermal Rayleigh number, $v=\frac{\mu}{p_{0}}$ is the kinematic viscosity, $C=\frac{\mu_{c}}{\mu d^{2}}$ is couple stress parameter, $Q$ is the heat source which defined as $Q=\frac{q d^{2}}{2 \kappa \Delta T}, \xi=\frac{K_{x}}{K_{z}}$ and $\eta=\frac{\kappa_{T x}}{\kappa_{T z}}$ are the mechanical and thermal anisotropy parameter respectively.

Besides, linear stability analysis is applied from Eq. (13) and Eq. (14) in order to eliminate the nonlinear term on the system. Normal mode expansion has been used for the resulting equations which defined respectively as below

$$
\left(w, D^{2} W, \Theta\right)=(W(z), \Theta(z)) \exp \left[i\left(a_{k}+a_{l}\right)+\sigma t\right],
$$

to get the equation as follows

$$
\begin{aligned}
& {\left[\frac{\sigma}{V a}\left(D^{2}-a^{2}\right)+\left(\frac{D^{2}}{\xi}-a^{2}\right)\left(1-C\left(D^{2}-a^{2}\right)\right)\right] W+a^{2} R a \Theta=0,} \\
& \left(D^{2}-\eta a^{2}-\sigma\right) \Theta+(1-Q(1-2 z)) W=0,
\end{aligned}
$$

where $D=\frac{d}{d z}$ and $a^{2}=a_{k}^{2}+a_{l}^{2}$. and the horizontal $a_{x}, a_{y}$ at direction of $x$ and direction of $y$ defined as the horizontal wave number with $\sigma$ represents the growth rate, respectively. The boundary conditions for this problem are given by

$W=D^{2} W=\Theta=0$ at $z=0$ and 1.

For Vadasz number, it is only valid for oscillatory state convection while stationary convection which remain the same during the Rayleigh number for the onset of convection occured at stable steady state, as verified by Srivastava et al., [12]. For this paper, stationary convection has been considered with couple stress fluid and internal heating effects in porous medium. By using Chevyshev Tau method with QZ algorithm, Eq. (16) and Eq. (17) has been solved numerically subject to upper free conducting boundary condition in Eq. (18). The interval form from the map has been 
transformed from $z \in[0,1]$ map into $x \in[-1,1]$ by using the equation of $x=2 x-1$ and the equation depict

$\frac{\partial}{\partial z}=2 \frac{\partial}{\partial x}=D, \quad x \in[-1,1]$

The variables equations in the expansion of Chebyshev polynomial in the form of expansion of

$y_{r}(x)=\sum_{k=0}^{M=1} a_{k r} T_{k}(x), \quad 1 \leq r \leq 6$,

where $T_{k}(x)$ is the Chebyshev polynomials with first order of $k$. The variables $y_{r}$ has been transformed as follows

$y_{1}=W, \quad y_{2}=D W, \quad y_{3}=D^{2} W \quad y_{4}=D^{3} W, y_{5}=\Theta, \quad y_{6}=D \Theta$.

Then Eq. (21) will substitute into Eq. (16) and Eq. (17) with the boundary conditions in Eq. (18). Ordinary differential equations obtained together with the boundary conditions and the eigenvalue problem arranged as given

$\frac{d Y}{d x}=L Y+\sigma J Y$

where $L$ and $J$ are real matrix with the order of 6. Eq. (21) reduced into $E X=\sigma F X$ where $E$ and $F$ are matrices of the block forms with boundary conditions included in the matrix. The system can be solved by using QZ algorithms in FORTRAN programming.

\section{Results}

In this paper, Chebyshev Tau method has been used to acquire the numerical solution of the ordinary differential equations in Eq. (16) and Eq. (17) with boundary conditions in Eq. (18). Linear stability analysis also been used and the eigenvalue results is obtained with QZ algorithms. All the parameters are observed along with the effects on Rayleigh number, $R a$ and will presented graphically to show how the parameters react when Rayleigh number, $R a$ opposed to the wave number from Figure 2 until Figure 5, respectively. From Figure 2 until Figure 5, the wave number, $a$ start from 1 because we want to focus on the point of the graph. Moreover, the critical Rayleigh number, $R a_{c}$ also will be plotted and displayed on Figure 6 until Figure 8.

First, Figure 2 presented the Rayleigh number, $R a$ against wavenumber, $a$ with distinct values of anisotropic parameter, $\eta$ for the fixed value when $\xi=0.5, Q=2$ and $C=2$. From the graph, increasing the value of $\eta$ will lead the value of $R a$ to increase too. Therefore, with the presence of anisotropic porous medium, $\eta<2$, the onset of convection will be delayed. Figure 3 displayed the neutral stability curve of Rayleigh number, $R a$ versus wave number, $a$ for various values of mechanical anisotropic parameter, $\xi$ at a constant value of $\eta=0.5, Q=2$ and $C=2$. It is shown on the graph, the increasing number of $\xi$ will lead to decrease in $R a$ values. The stability convection in porous medium is delayed at $\xi=1.2$. This was due the movement of the heat upward and will increase the chance of $\xi$ to react on the porous medium. Thus, the effect of increasing $\xi$ will destablize the system. 
The influenced of internal heat parameter in porous medium on the value of $R a$ at fixed value of $\xi=0.5, \eta=0.5$ and $C=2$ against wave number, $a$ shown in Figure 4 . The observation on the graph shows $R a$ increasing with decreasing value of internal heat parameter, $Q$. Therefore, the internal heat parameter has the effect of destablizing the system on stability convection. Figure 5 shows the graph of $R a$ versus $a$ with various values of couple stress parameter, $C$ with respect to $\eta=0.5, Q=2$ and $\xi=0.5$ as constant values. From the figure, it shows that when the Rayleigh number, $R a$ increases, the couple stress paramerer, $C$ will increase too. Thus, it will delay the onset of convection in the system. As a result, couple stress parameter will also stablizing the system of convection in the porous medium.

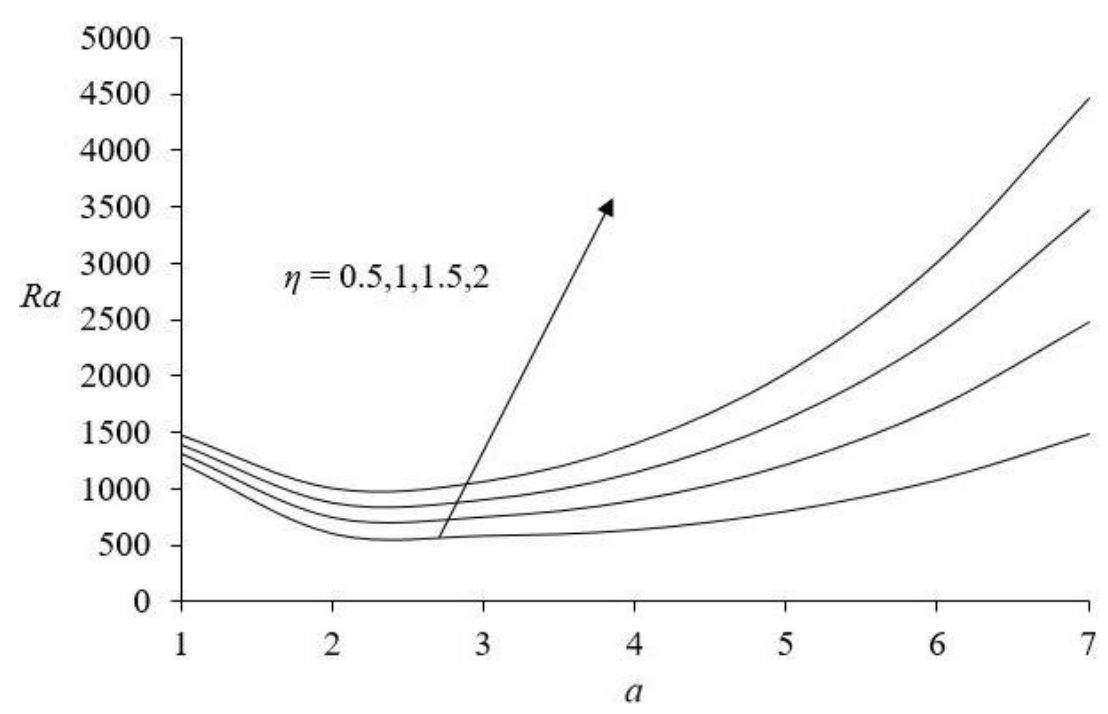

Fig. 2. The neutral stability curve, $R a$ versus $a$ for different values of $\eta$

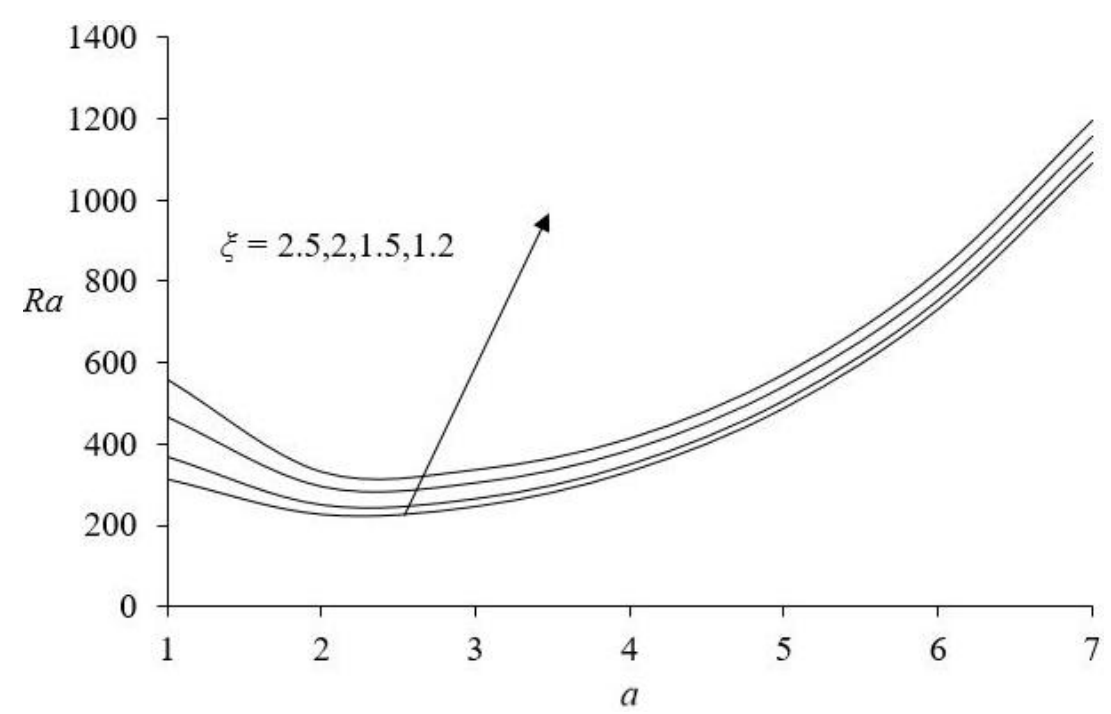

Fig. 3. The neutral stability curve, $R a$ versus $a$ for different values of $\xi$ 


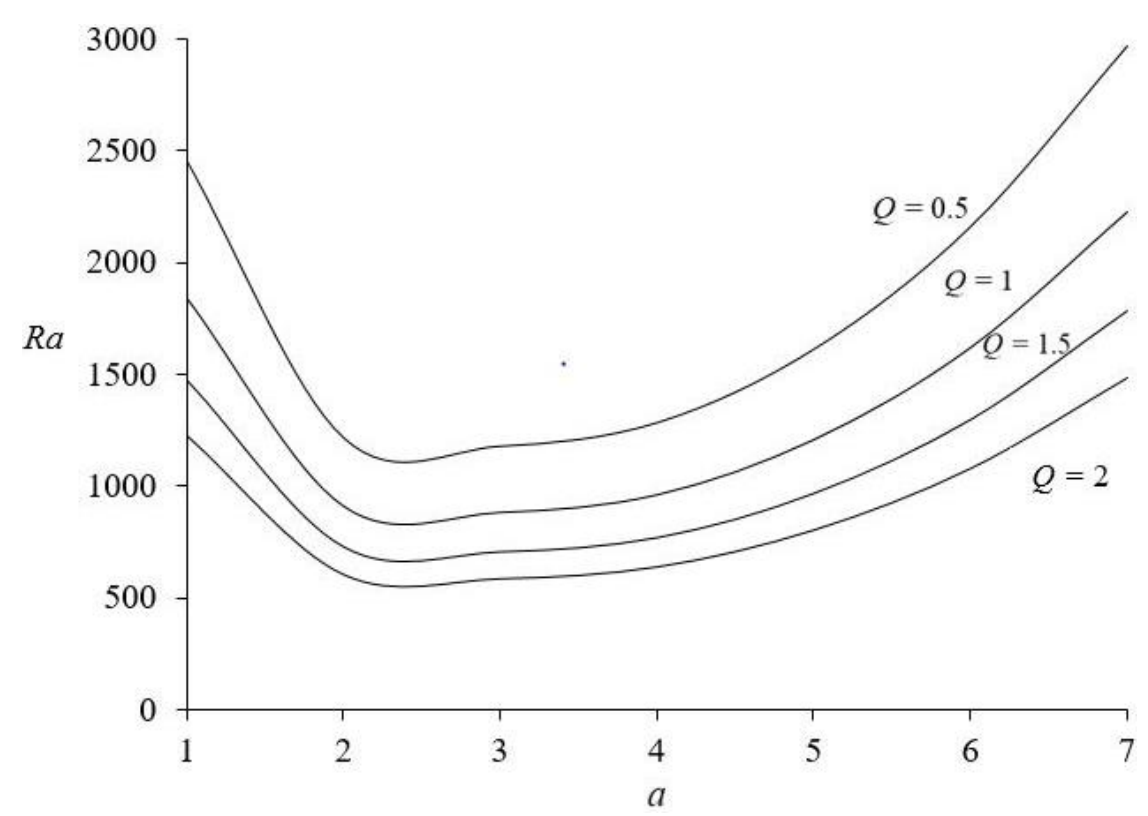

Fig. 4. The neutral stability curve, $R a$ versus $a$ for different values of $Q$

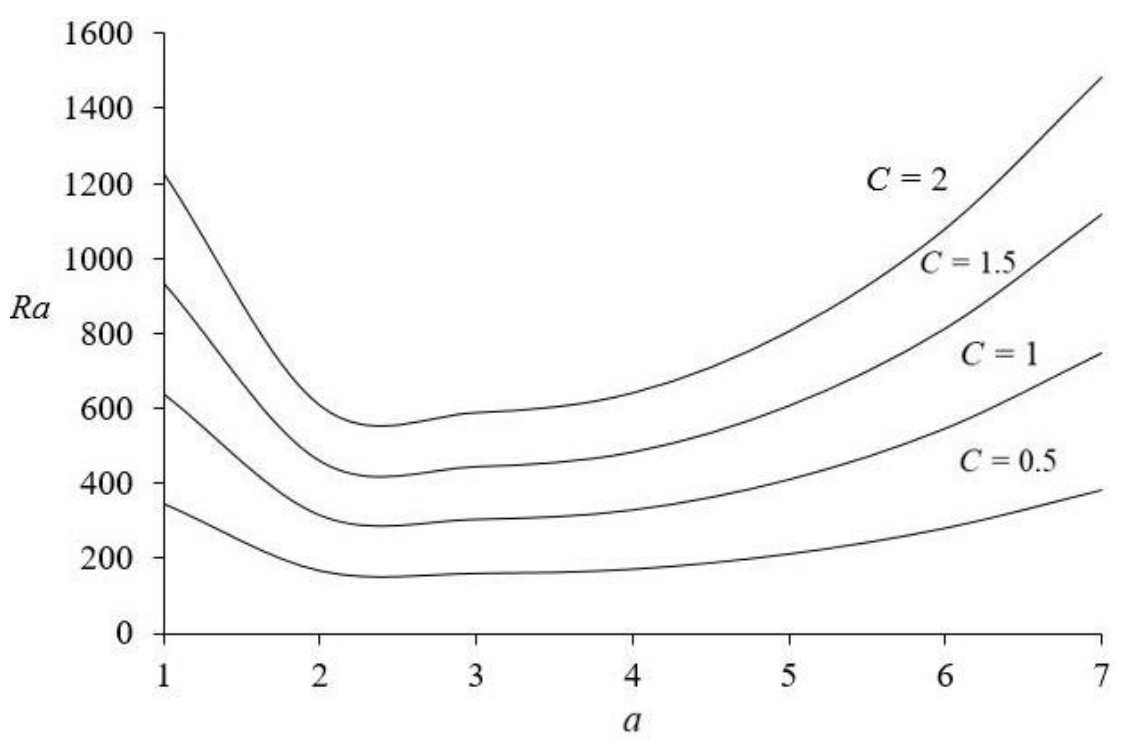

Fig. 5. The neutral stability curve, $R a$ versus $a$ for different values of $C$

Furthermore, the critical Rayleigh number, $R a_{c}$ which supported minimal value of the Rayleigh number has been obtained and plotted in the Figure 6 until Figure 8, respectively. The relation between $Q, \eta, \xi$ and $C$ is calculated and has been displayed in the figure. Figure 6 represented the point where the critical Rayleigh number, $R a_{c}$ in porous medium with couple stress parameter, $C$ for different values of $\eta$. From Figure 6 , the values of $R a_{c}$ increases when $C$ increases, respectively. The results obtained has a good deal with Malashetty and Kollur [4] and thus verified our analysis when $\eta$ also increases. 


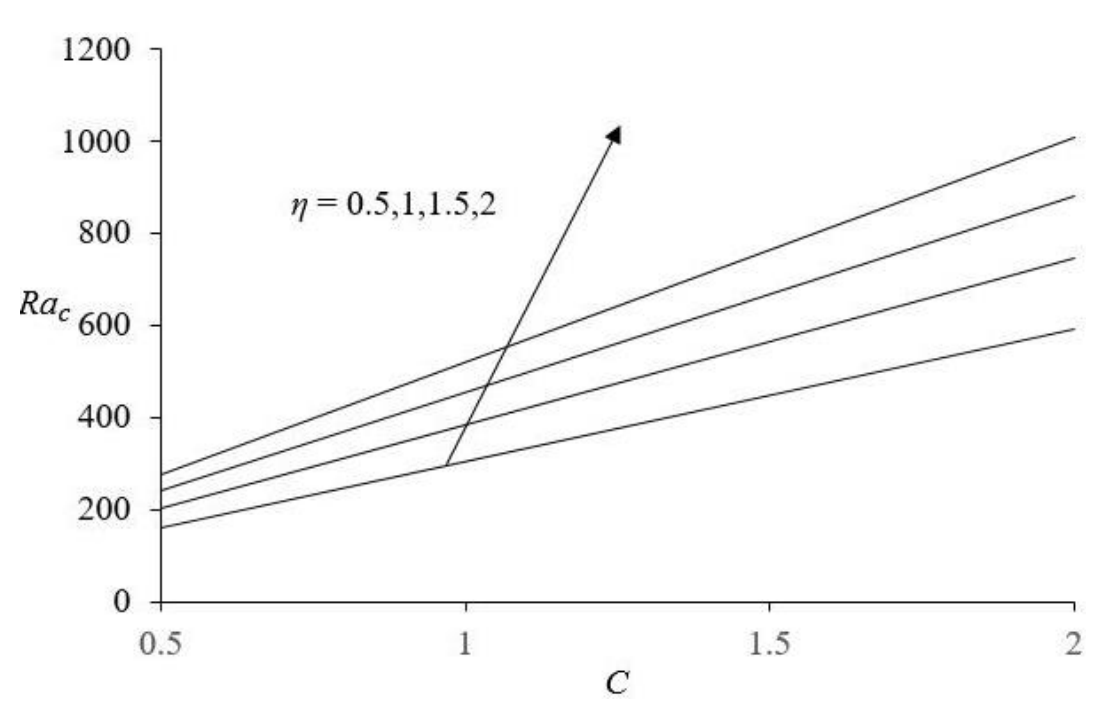

Fig. 6. The critical Rayleigh number, $R a_{c}$ versus $C$ for different values of $\eta$

The graph of critical Rayleigh number, $R a_{c}$ inside the porous medium against mechanical anisotropic parameter, $\xi$ with dissimilar values of couple stress parameter, $C$ depicted in Figure 7 . From the figure, the values of $R a_{c}$ decreases when $C$ decreases, respectively. Figure 7 indicate the critical Rayleigh number, $R a_{c}$ is approaching the minimum value when mechanical anisotropic parameter, $\xi$ is increasing. Thus, it is verified the system is stablized with the presence of couple stress. Next, Figure 8 also shows the critical Rayleigh number, $R a_{c}$ in porous medium versus internal heating parameter, $Q$ with various values of couple stress parameter, $C$ when $\xi=0.5$ and $\eta=0.5$. It is observed that when the internal heat parameter, $Q$ increases, the critical Rayleigh number, $R a_{c}$ will decreases. This will make the effect of source of the internal heat parameter destablize the start of stability convection, as verified by Srivastava et al., [12].

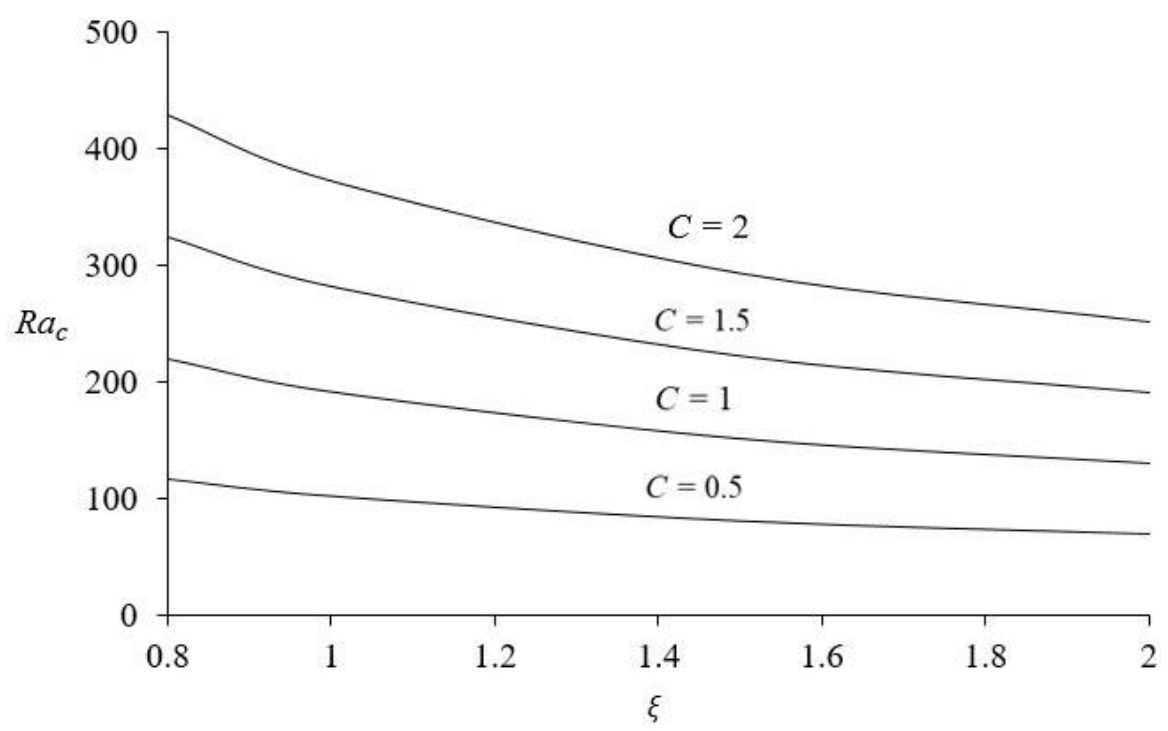

Fig. 7. The critical Rayleigh number, $R a_{c}$ versus $C$ for different values of $\xi$ 


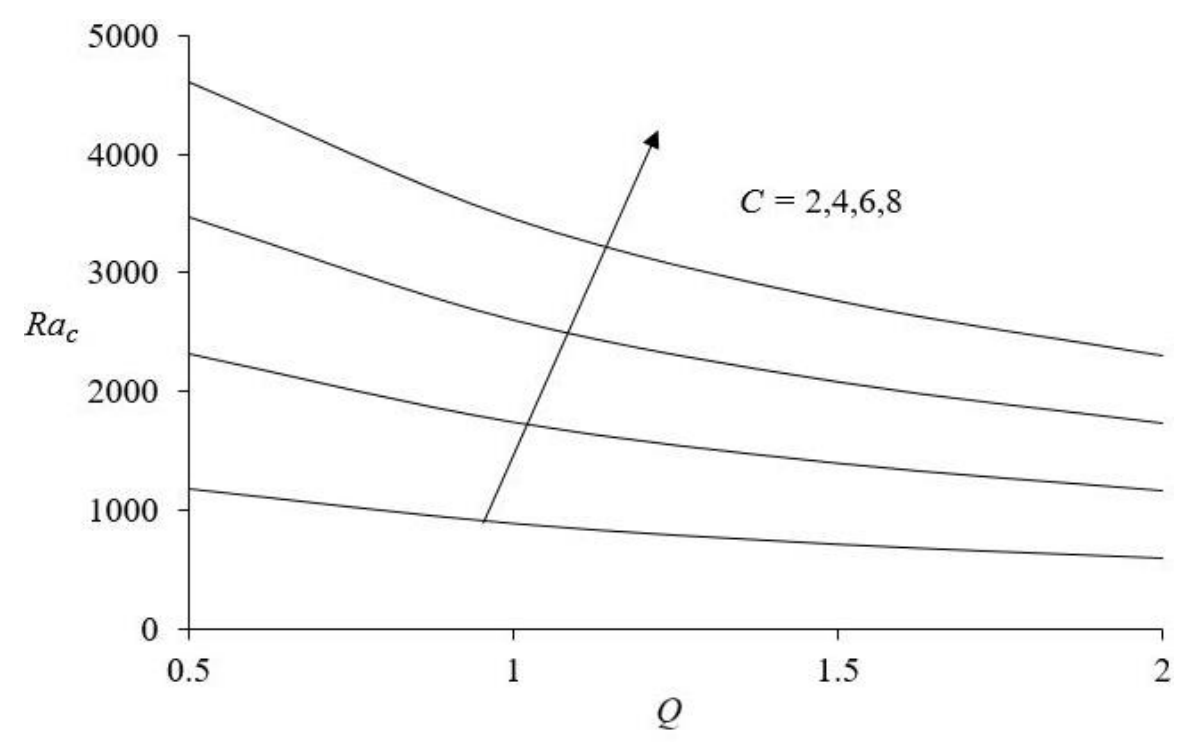

Fig. 8. The critical Rayleigh number, $R a_{c}$ versus $Q$ for different values of $C$

\section{Conclusions}

Couple stress fluid with stability convection horizontal saturated anisotropic porous medium in the presence of internal heating has been studied analytically by using linear stability analysis. The eigenvalue problem is solved by using Chebyshev Tau method with QZ algorithm. In this study, the combine effect of the couple stress fluid and internal heating also has been concerned. The critical Rayleigh number, $R a_{c}$ increases when the effect of couple stress fluid occurred and decreases when the internal heating effect found in the system. Therefore, from the result obtained, thermal anisotropic parameter, $\eta$ and couple stress fluid, $C$ act as stablizer on the system while internal heating, $Q$ and mechanical anisotropic parameter, $\xi$ act as destablizer on the system.

\section{Acknowledgement}

The present research is supported by the Putra Grant - Putra Graduate Initiative (IPS) -GPIPS/2018/9642900.

\section{References}

[1] Bhadauria, B. S. "Double-diffusive convection in a saturated anisotropic porous layer with internal heat source." Transport in Porous Media 92, no. 2 (2012): 299-320. https://doi.org/10.1007/s11242-011-9903-x

[2] Stokes, Vijay Kumar. "Couple stresses in fluids." The Physics of Fluids 9, no. 9 (1966): 1709-1715. https://doi.org/10.1063/1.1761925

[3] Gaikwad, S. N., and S. Kouser. "Double diffusive convection in a couple stress fluid saturated porous layer with internal heat source." International Journal of Heat and Mass Transfer 78 (2014): 1254-1264. https://doi.org/10.1016/i.ijheatmasstransfer.2014.07.021

[4] Malashetty, M. S., and Premila Kollur. "The onset of double diffusive convection in a couple stress fluid saturated anisotropic porous layer." Transport in Porous Media 86, no. 2 (2011): 435-459. https://doi.org/10.1007/s11242-010-9630-8

[5] Sharma, R. C., and Mohinder Pal. "On a couple-stress fluid heated from below in a porous medium in the presence of a magnetic field and rotation." Journal of Porous Media 5, no. 2 (2002). https://doi.org/10.1615/JPorMedia.v5.i2.70

[6] Pranesh, S., and Ritu Bawa. "Effects of Magnetic Field and Internal Heat Generation on the onset of Rayleigh-Bénard Convection in Nanofluid." International Journal of Engineering Research \& Technology 4 (2015). https://doi.org/10.17577/IJERTV4IS050063 
[7] Nield, D. A., and A. V. Kuznetsov. "Onset of convection with internal heating in a porous medium saturated by a nanofluid." Transport in Porous Media 99, no. 1 (2013): 73-83.

https://doi.org/10.1007/s11242-013-0174-6

[8] Storesletten, Leiv, and D. Andrew S. Rees. "Onset of convection in an inclined anisotropic porous layer with internal heat generation." Fluids 4, no. 2 (2019): 75.

https://doi.org/10.3390/fluids4020075

[9] Srivastava, Alok, B. S. Bhadauria, P. G. Siddheshwar, and I. Hashim. "Heat transport in an anisotropic porous medium saturated with variable viscosity liquid under g-jitter and internal heating effects." Transport in Porous Media 99, no. 2 (2013): 359-376.

https://doi.org/10.1007/s11242-013-0190-6

[10] Malashetty, M. S., and Rajashekhar Heera. "The effect of rotation on the onset of double diffusive convection in a horizontal anisotropic porous layer." Transport in Porous Media 74, no. 1 (2008): 105-127. https://doi.org/10.1007/s11242-007-9183-7

[11] Bhadauria, B. S., Anoj Kumar, Jogendra Kumar, Nirmal C. Sacheti, and Pallath Chandran. "Natural convection in a rotating anisotropic porous layer with internal heat generation." Transport in Porous Media 90, no. 2 (2011): 687. https://doi.org/10.1007/s11242-011-9811-0

[12] Srivastava, Alok, B. S. Bhadauria, and I. Hashim. "Effect of internal heating on double diffusive convection in a couple stress fluid saturated anisotropic porous medium." Advances in Materials Science and Applications 3, no. 1 (2014): 24-45.

https://doi.org/10.5963/AMSA0301004 